\title{
Mixed Integer Linear Programming Approach to Optimize the Hybrid Renewable Energy System Management for supplying a Stand-Alone Data Center
}

\author{
Marwa Haddad, Jean Marc Nicod*, Christophe Varnier* and Marie-Cécile Péra ${ }^{\dagger}$
}

February 11, 2020

\begin{abstract}
The trend toward server-side computing and the exploding popularity of Internet services due to the increasing of demand for networking, storage and computation has created a world-wild energetic problem and a significant carbon footprint. These environmental concerns prompt to several green energy initiative aiming either to increase data center efficiency and/or to the use of green energy supply. In this regard, As part of the ANR DATAZERO project, many researchers are working to define main concepts of an autonomous green data center only powered by renewable energies. Thus, the present paper proposes a mixed integer linear program to optimize the commitment of a hybrid energy system composed of wind turbines, solar panels, batteries and hydrogen storage systems. The approach is used to supply a data center demand and takes the weather forecasts into account at the time of optimization. Different time window resolution are applied in order to verify the best time window for decision making.
\end{abstract}

Keywords - Renewable energy supply; Operational research; Integer linear programming; Hybrid energy system; Energy management; Optimization

\section{Introduction}

The computing capacity and the number of servers in data centers are more and more increasing to meet the soaring load demand of IT services, applications and storage. The high power consumption has led to many serious consequences such as increasing energy demand which has pushed the electrical power to enter a new evolution phase. It can be mainly identified by increasing interest about climate change, by a transition to a green renewable energy economy, as well as efficient use of energy (1).

In this context, this paper considers the use of renewable energy to displace fossil fuels. It consists in optimizing the management of a hybrid energy system composed of photovoltaic panels, wind turbines, battery storage system and a hydrogen storage system in order to supply the data center demand all over its life time. Moreover, latest research works on power management aimed at reducing data centers power consumption, are actually divided into two strategies. The first one consists in reducing the consumption of computing devices using different hardware and software technologies including workload scheduling (7), virtual marchine (VM) management (17), cooling computing power balancing (3), etc. The second strategy, the one on which this paper takes part consists in optimizing the design of urban settlements by means of the exploitation of natural sources of energy and the development of building management systems (13) in order to make an efficient use of electrical energy.

Among many alternative energy sources used, solar and wind are considered as an attractive solution (12) to generate power on a large scale and are widely used for substituting fossil fuels in the electrical power process. However, due to their intermittent and variable nature, the integration of such sources is a challenge to reach a low-carbon society

${ }^{*}$ FEMTO-ST Institute, Univ. Bourgogne Franche-Comté / CNRS / ENSMM / UTBM, F-25000 Besançon, FRANCE

${ }^{\dagger}$ FCLAB, FR 3538, Univ. Bourgogne Franche-Comté / CNRS, , F-90010 Belfort, FRANCE 
as it needs storage to enable satisfaction of the demand. Then, it is quite necessary to integrate multiple energy sources working together (batteries, biomass, wind, solar, hydrogen, geothermal) as the balance between generation and demand must be met in real-time (14). This is a common concern for both small/big power systems to can operate islanded, as it needs an optimal management of the storage system (11). Nevertheless, nowadays, research tends to build bigger standalone buildings or datacenters $100 \%$ powered by green energy (9).

This paper presents, as part of the ANR DATAZERO 11 research project, an effective management of a hybrid renewable energy system (HRES) in order to completely supply a standalone data center. We propose here a model based on Mixed Integer Linear Programming approach to optimize the commitment of energy sources. This is done in compliance with user demand with level maximization long-term energy stocks. This approach takes into account the season and forecasts weather when making decisions. For a better understanding of the models, a table of the notation used is presented in Table 1 .

The remainder of this article is organized as follows: Section 2 describes the models of energy sources used in the optimization problem. Then, in section 3 the problem statement is explained and solved. Section 4 define the metric used to evaluate the obtained solutions and Section 5 illustrates different use case with the presentation of a solution. The paper ends with a conclusion and perspectives.

\section{Model of a hybrid renewable energy system}

The power system of the data center is composed of solar panels $(p v)$, wind turbines $(w t)$, fuel cells $(f c)$, batteries, electrolysers, hydrogen tanks and other appliances of energy conversion. In order to analyze and properly engage the hybrid power system, it is necessary to understand the pattern of each component. In the following, we consider an $\mathcal{H}$ horizon discretized into $K$ periods of $\Delta t$ units of time such that $\mathcal{H}=K \Delta t$. We assume that during a period $\Delta t$, for all $t$ with $k \Delta t \leq t<(k+1) \Delta t$ and $k \in \llbracket 0, K-1 \rrbracket$, the parameters and variables of the model are constant.

The modeling of the components is described in the following according to the parameters of the system and the time step $k$ of the discrete work horizon $\mathcal{H}$ :

\subsubsection{Wind Turbine Generator}

Dong et al. (5) and many other researchers have used the same mathematical model for the output power $P w t_{k}$ of the wind turbine. The same model will be used in our study.

\subsubsection{Photovoltaic Panel}

To modelize the output power of PV panels $P p v_{k}$, a widely used model (16) assumes that the irradiance is proportional to the surface of the panels.

\subsubsection{Batteries}

The batteries are used for short term storage. The state of charge of the batteries is calculated for each $k$ with $k \in \llbracket 1, K \rrbracket$ with respect to the previous state of charge of the battery $S O C_{k-1}$, the self-discharge rate $\sigma$, the charging and discharging power $P c h_{k-1}, P d c h_{k-1}$, the charging and discharging efficiency $\eta_{c h}, \eta_{d c h}$ as follows:

$$
\begin{aligned}
S O C_{k} & =S O C_{k-1} \times(1-\sigma)+P c h_{k-1} \times \eta_{c h} \times \Delta t \\
& -\frac{P d c h_{k-1}}{\eta_{d c h}} \times \Delta t
\end{aligned}
$$

As the battery storage system (BSS) cannot charge and discharge simultaneously, the value of $S O C_{k}$ is bounded by the minimum amount of $S O C$ min and maximum energy $S O C \max$. So $\forall k \in \llbracket 1, K \rrbracket$ with $S O C_{0}=S O C_{i n i t}$ :

$$
S O C_{k}=\min \left\{S O C_{k-1} \times(1-\sigma)+P c h_{k-1}\right.
$$

1 http://www.datazero.org 


$$
\begin{aligned}
& \left.\times \eta_{c h} \times \Delta t, S O C \max \right\} \text { if } P c h_{k-1}>0 \\
S O C_{k} & =\max \left\{S O C_{k-1} \times(1-\sigma)-\frac{P d c h_{k-1}}{\eta_{d c h}}\right. \\
& \times \Delta t, S O C \min \} \text { if } P d c h_{k-1}>0
\end{aligned}
$$

\subsubsection{Electrolyzer}

The operating power of the electrolyzer $P e z_{k}$ depends of the hydrogen mass flow $Q e z_{k}$ in $(k g)$, and is bounded by Pezmin, Pezmax the operating range of the electrolyzer. This relation is defined for each time step $k(k \in \llbracket 0, K-1 \rrbracket)$, with $\eta_{e z}$ the electrolyzer efficiency and $H H V h_{2}$ the hydrogen higher heating value, as:

$$
\begin{aligned}
P e z_{k} \times \Delta t & =\frac{H H V h_{2} \times Q e z_{k}}{\eta_{e z}} \\
\text { with } P e z \min & \leq P e z_{k} \leq \text { Pezmax }
\end{aligned}
$$

\subsubsection{Fuel Cell}

The hydrogen mass flow $Q f c_{k}$ depends of the output power of the fuel cell $P f c_{k}$ at any time during each period $k$ $(k \in \llbracket 0, K-1 \rrbracket)$ as in Equation $\left(5 \rrbracket\right.$ with $\eta_{f c}$ the efficiency and $L H V h_{2}$ the low heating value of hydrogen:

$$
\begin{array}{r}
P f c_{k} \times \Delta t=L H V h_{2} \times Q f c_{k} \times \eta_{f c} \\
\text { with } \quad P f c_{k} \leq P f c \max
\end{array}
$$

\subsubsection{Hydrogen tank}

The level of hydrogen produced by the electrolyzer and consumed by the fuel cell in the tank is determined at any time during each period $k$ with $k \in \llbracket 1, K \rrbracket$ as follows:

$$
\begin{gathered}
L O H_{k}=L O H_{k-1}+Q e z_{k-1}-Q f c_{k-1} \\
\text { with } 0 \leq L O H_{k} \leq L O H \max
\end{gathered}
$$

with $\mathrm{LOH}_{0}$ the initial value of the level of hydrogen.

The previous models for each energy/power source are used to solve the tackle optimization problem.

\section{Problem statement and resolution}

The targeted data center is intended to be used all year long without resorting to the conventional power grid. In order to make it possible, the latter should maintain an energy level in its long-term storage in order to be able to operate even if days where the power from renewable sources is not enough to address the power demand. Indeed, the idea is that the most favorable days (let say in summer) has to compensate for the worst days (let say in winter). The challenge is therefore to smooth consumption over the year thanks to long-term storage $\left(\mathrm{H}_{2}\right)$ and over days thanks to the short term storage (batteries). For the takled problem, we agree that a study of meteorological data over several years can predict what the level of hydrogen stocks should be at a macroscopic point of view. It is then a question of scheduling a commitment of the sources, and thus managing both the short term and long term storage, in order to stick to these forecasts.

The optimization problem is then to maximize the power production $\operatorname{Pprod}_{k}$ with maintaining the level of hydrogen $L O H_{K}$ at the end of the horizon $\mathcal{H}$ to make possible the seasonal compensation without wasting energy and to be able to handle the next season. The following sections describe constraints to respect the energy component usage and the data center power demand. 


\subsection{Flow conservation}

In order to maintain a better use of the sources, the renewable energy produced $\left(P w t_{k}+P p v_{k}\right)$ by wind turbines and photovoltaic panels during the whole time step $k$ is used for three purposes:

- Satisfying of the data center load $\left(\right.$ Pload $\left._{k}\right)$;

- Charging the hydrogen tanks thanks to electrolyzers $\left(P e z_{k}\right)$;

- Charging the batteries $\left(P c h_{k}\right)$.

In case of inability to produce enough renewable energy to meet the data center demand $\left(P l o a d_{k}\right)$, additional electrical power is delivered by the batteries $\left(P d c h_{k}\right)$ and fuel cells $\left(P f c_{k}\right)$. This use can be mathematically written by Equation $(8)$ for each $k \in \llbracket 0, K-1 \rrbracket$ :

$$
\begin{aligned}
\text { Pload }_{k} & \leq P w t_{k}+P p v_{k}+ \\
& \left(P f c_{k}+P d c h_{k}-P e z_{k}-P c h_{k}\right) \times \eta_{i n v}
\end{aligned}
$$

\section{$3.2 \quad$ Mutual exclusion rules}

Common sense rules have to allow the use of power components without any time restriction if the two following constraints are respected:

- When fuel cells are operating, it is only used to meet the demand and not to charge batteries.

- When batteries discharge, they are only used to meet the demand and not to produce hydrogen.

\subsection{Mathematical Model}

The resulting model can be viewed as a Constraint Satisfaction Problem (CSP) as a mathematical program that can be linearized as a Mixed Integer Linear Program (MILP) presented in the next section. The reader can find more explanations on the linearization in (10). So we can optimally solve the addressed problem using an solver as Gurobi (8). This allows to propose solutions by the Power module of the project: (i) identification of the profile that maximizes the power produced, (ii) computation of an optimal commitment around a target profile considering a given relax factor, and (iii) computation of an optimal commitment constrained by the load profile.

Therefore, the CSP is defined as follows (with $k \in \llbracket 0, K-1 \rrbracket$ or $k \in \llbracket 0, K \rrbracket$ for $S O C_{k}$ and $L O H_{k}$ variables):

$$
\begin{aligned}
& \text { (8), 2), (3), (4), (5), (6) } \\
& \text { Bounds: } \\
& P f c_{k} \leq P f c m a x \quad \forall k \in \llbracket 0, K-1 \rrbracket \\
& \text { Pezmin } \leq \text { Pez } z_{k} \leq \text { Pezmax } \forall k \in \llbracket 0, K-1 \rrbracket \\
& S O C \min \leq S O C_{k} \leq S O C \max \quad \forall k \in \llbracket 0, K \rrbracket \\
& 0 \leq L O H_{k} \leq L O H \max \quad \forall k \in \llbracket 0, K \rrbracket
\end{aligned}
$$

\subsection{Obtained linear program}

In order to be solved, the proposed CSP (9) was linearized following (2). The obtained linear program is detailed as in Equation 10 . 
The objective function consist in maximizing the power production Pprod $_{k}$ hour by hour to increase the chance to fulfill the data center power demand Pload $_{k}$.

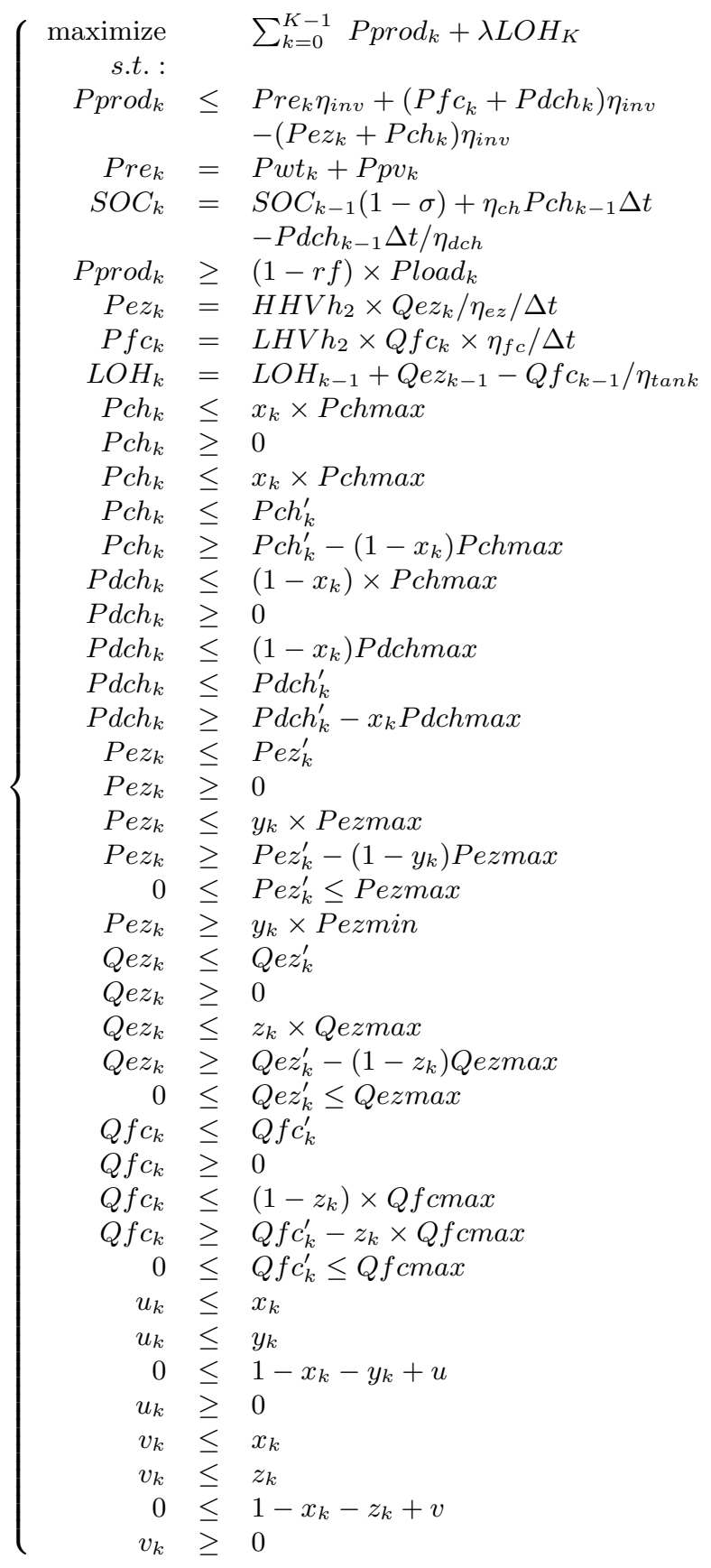




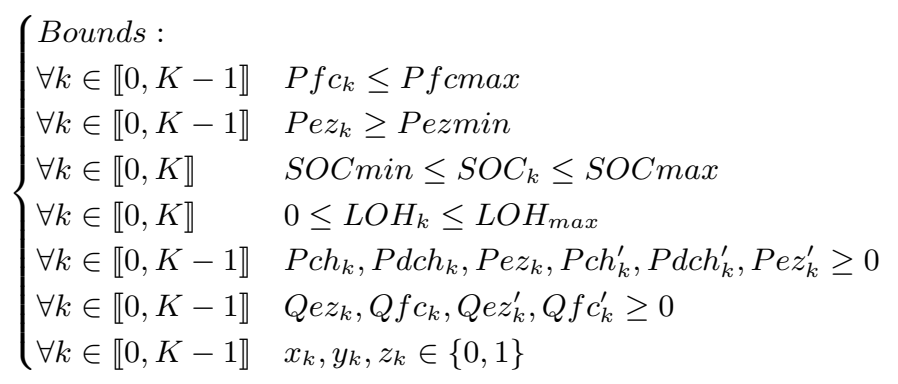

Moreover, the long term storage $\mathrm{LOH}_{K}$ should be strongly maintained rather than allowing waste if overproduction is too high. Thus, a high value for $\lambda$ is necessary to offset the fact that some of the energy produced is lost due to electrolyser and fuel cell efficiency.

Also, in order to match Pprod $_{k}$ with the data center load Pload $k$ for each time step $k$, a relax factor $r f$ is used as in the constraint 11 .

$$
\operatorname{Pprod}_{k} \geq(1-r f) \times \text { Pload }_{k}
$$

In case of no existing solutions due to power production deficiency to supply the data center, the relax factor value between $0 \leq r f \leq 1$ is computed using a binary search approach in order to degrade the power response for the data center as less as possible. As the data center load is relaxed, another management is proposed. The solution is thus sub-optimal compared to the initial power demand, but a alternative feasible solution is proposed to overcome the intermittent nature of renewable sources. The evaluation of this degradation impact is evaluated in Section 5

The obtained model used to provide a power profile that can match as close as possible with a power demand is the MILP (10) (We recall that additional explanations are available in (10)):

\section{Metrics}

In order to evaluate the solutions obtained by the MILP, some metrics are explained in this section:

\subsection{Reliability}

Due to the intermittent nature of the power produced by PV and WT, analyzing the reliability of the system is quite important. Actually, an electrical system is considered reliable when it is able to supply the required power. There are many methods for carrying out the reliability analysis: The Loss of Power Supply Probability (LPSP) defined in the following is one of the most used ones:

$$
L P S P=\frac{\sum_{k=0}^{K-1} \Delta t\left(\text { s.t. } \text { Pprod }_{k}<\text { Pload }_{k}\right)}{\mathcal{H}}
$$

where $\mathcal{H}$ is the number of hours of the horizon. A LPSP equals to 0 means that the system is absolutely reliable and a LPSP equals to 1 means that the demand is not satisfied at all by the generated power.

\subsection{Level of Autonomy (LA)}

The Level of Autonomy (LA) is defined as the percentage of time where the demand is satisfied by the renewable energy solely. It is computed as defined in Equation (13).

$$
L A=\frac{\sum_{k=0}^{K-1} \Delta t\left(\text { s.t. } \text { Pre }_{k} \geq \text { Pload }_{k}\right)}{\mathcal{H}} \times 100
$$




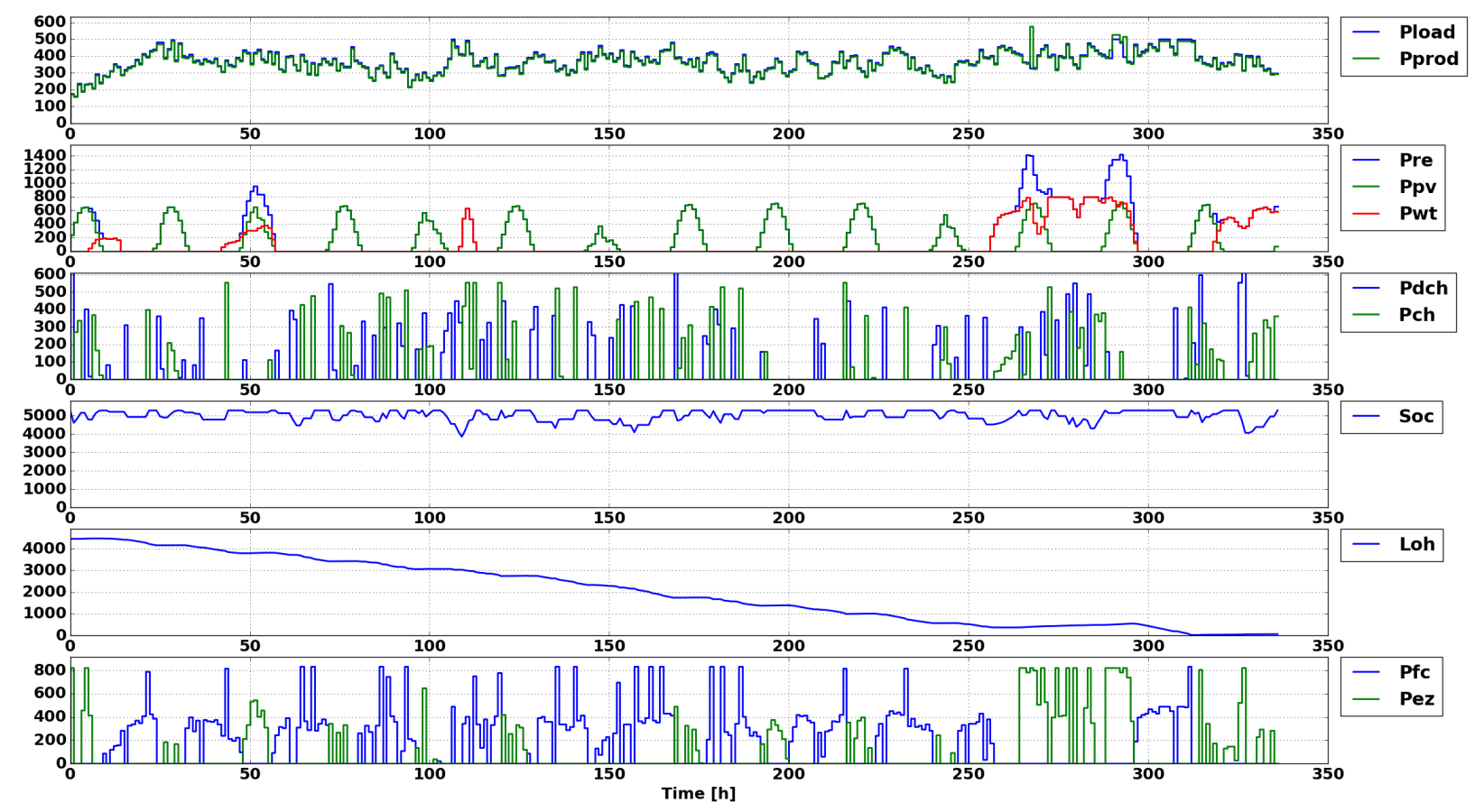

Figure 1: Management of the renewable sources for 15 days with $r f=0.02$

\subsection{Unused renewable energy (URE)}

As the charging and discharging power of both system batteries and hydrogen are constants, the MILP enable, sometimes, to use all the energy produced by the renewable sources. Thus, this quantity of unused renewable energy is computed as:

$$
U R E=\sum_{k=0}^{K-1}\left(\frac{\max \left(\operatorname{Prod}_{k}-\text { Pload }_{k}, 0\right) \times \Delta t}{\mathcal{H}}\right)
$$

\subsection{Percentage of the Energy Produced to demand (PEP)}

It consists in computation of amount power produced Pprod $_{k}$ divided by the demand. This is mathematically proceeded by the following expression:

$$
\text { PEP }=\frac{\sum_{k=0}^{K-1} \min \left(\text { Pprod }_{k}, \text { Pload }_{k}\right)}{\sum_{k=0}^{K-1} \text { Pload }_{k}}
$$

\section{$5 \quad$ Experiments and discussions}

The Mixed Integer Linear Program is implemented using python and solved by GUROBI solver (8). The problem takes as inputs the weather conditions, power demand Pload $k$ and other information about the electrical power system sizing like number of wind turbines $N w t$, the solar panel surface $N p v$, battery storage capacity $S O C m a x$, the capacity of the hydrogen tank $\mathrm{H}_{2}$, the efficiency of each component. As an output, the solver gives a schedule of each time slot for supply-side source and energy storage usage, according to the availability of renewable power. The data representing the solar radiation and wind speed have been measured on an hourly scale from January 2004 
till December 2012 in a coastal area. To be more precise, the endogenous data of the solar radiation and wind speed time series have been measured at Los Angeles (Latitude: 34.57, Longitude: -118.02 , Time Zone: -8). These data has been obtained respectively from the National Solar Radiation DataBase (NSRDB) (15) and from wind prospector of the National Renewable Energy Laboratory (NREL) (므). From the IT part, the data center demand were obtained from the traces of access logs from the World Cup web site of 1998 (4). Based on these data, a sizing study was made and an average infrastructure as showed in the table 2 is given. This sizing is an input the MILP needed to be solved.

The same infrastructure sizing will be used in many management scenarios under different weather conditions for a better illustration of the difference between the obtained results, but first, we proceed to the verification of the proper functioning of the optimization strategy.

\section{$5.1 \quad$ Management results}

Figure 1 shows a solution obtained by solving the MILP on a given period $\mathcal{H}=14$ days $=336 h$ with a time slot $\Delta t=1 h$ in the winter during the year 2010. This year was chosen for a better illustration of the results. This solution consists of the evolution of each variable of the program, hour by hour, each visible on a specific window of Figure 1. The first windows shows the data center demand Pload and the produced power Pprod, used to supply the data center, optimized by the MILP. The second window shows in details the the solar panels Ppv, the wind turbines Pwt and the total Pre produced. The third window shows both the charging Pch and discharging power Pdch of the batteries followed by the variation of state of charge of the batteries $(S O C)$. The last two windows of Figure 1 show the actual level of hydrogen $L O H$ computed by the MILP. Finally, the electrolyzer power $P e z$ and fuel cell power $P f c$ obtained by the MILP is shown to explain the evolution of $\mathrm{LOH}$.

This figure was chosen in winter in the worst conditions of the year 2010 to show the aim of the relax factor. In fact, with an optimal relax factor $r f=0.02$, the power supplied to the data center is relaxed (i.e., reduced as less as possible) while a solution is possible because of the problem constraints. The first founded solution is then displayed. It is used in order to propose an alternative power profile if no solution exists to answer to the data center power demand. Thus, the latter could postpone or reschedule the jobs. Based on Figure 1. one can see that the battery state of charge comes back to its initial level each 24 hours because batteries are only responsible of short term energy storage (daily compensation) to ensure the hourly lack of renewable energy (e.g., solar night and day alternation) during the same day.

Also, as it is responsible of the seasonal offset, one can see that the level of hydrogen is decreasing as there is not enough renewable energy to ensure the demand (no wind) and get stabilized at the end of window.

Thus, as the linear program is working properly, the following subsection shows different usage of this program. Indeed, the management program should be able to find a solution under any weather conditions. The metrics explained in Section 4 are used to verify the robustness of our approach.

\subsection{Management scenarios results}

In this part, the program is executed following different resolution time windows for the same horizon (1 year) under different weather conditions. In fact, the same sizing will be played from the year 2004 till 2012. For each year, several resolution time windows are simulated with the weather condition of the year. One simulation consists in iteratively solving the power management program for a given period length $\mathcal{H}$ as many times as it is needed to cover the whole year. This resolution time window $\mathcal{H}$ is related to the weather prediction time. Different cases of resolution time window are exploited to shows the impact of the decision on the management proposed:

- 1st case: resolution window is equal to the horizon fixed (1 resolution for 1 year) 
- 2nd case: resolution window is equal to 1 week (52 resolutions for 1 year)

- 3rd case: resolution window is equal to 3 days (121 resolutions for 1 year)

- 4th case: resolution window is equal to 1 day (365 resolutions for 1 year)

The aim of this comparison is to choose the best management time window using this approach (Should the decision be taken only 1 time per week, per three days or per days?). The chosen metrics are applied on each cases study. For all cases, an average value of the metrics is computed and displayed in the following results. Experimentation are performed on an Intel@ Core $^{T M}$ i5-6200U CPU @ 2.30GHz $\times 4,8 \mathrm{~GB}$ RAM, 64-bit using Ubuntu 16.04 LTS as exploitation system. The solving time for each year with different resolution time windows will be also compared.

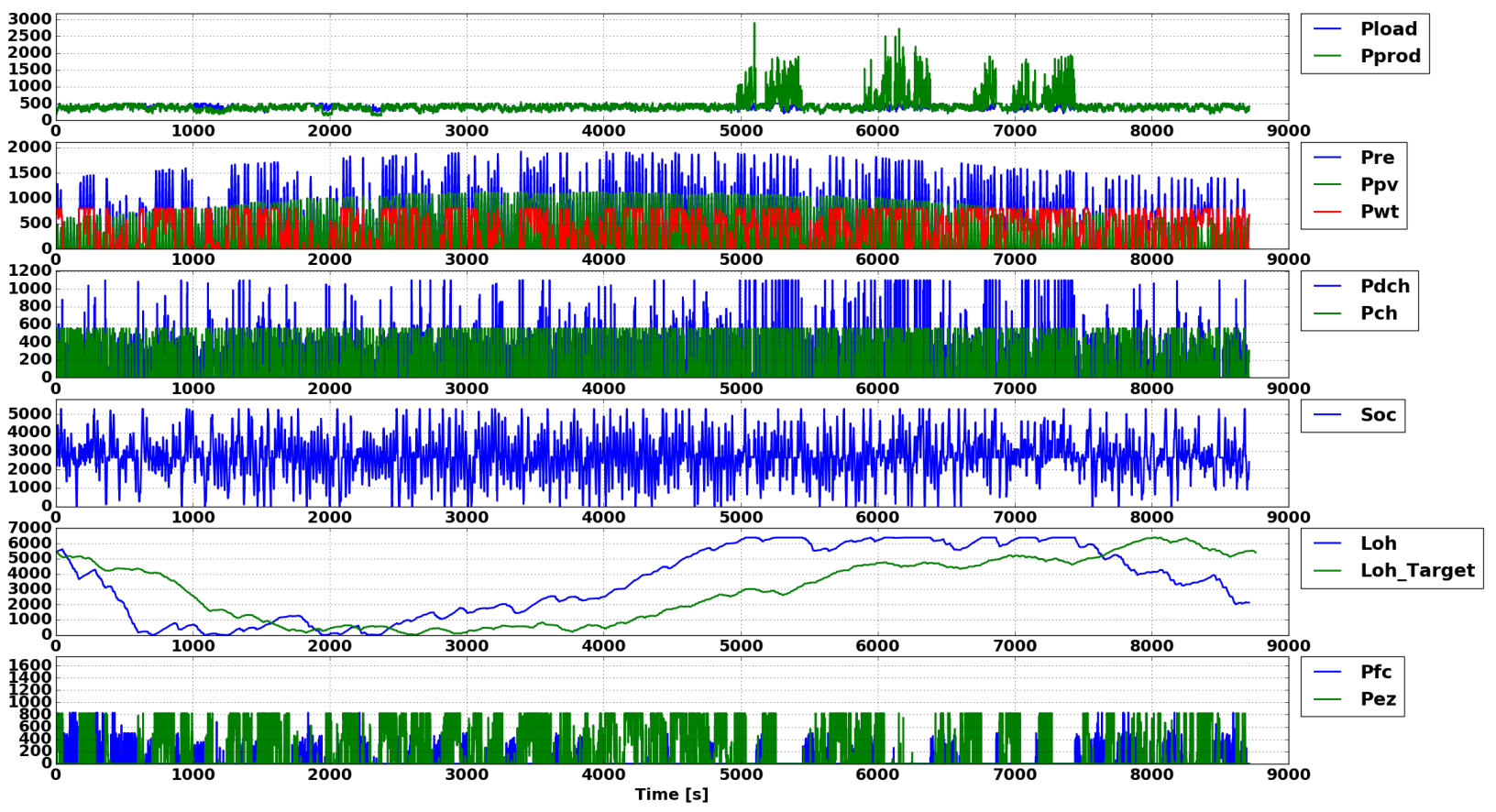

Figure 2: Management of the renewable sources in the 3rd case for the year 2008

In the following tables, the column LPSP is divided on two columns, the average and the standard deviation of the LPSP values obtained for each resolution in different simulations.

The table 3 resume the metric results for a one year resolution with $\mathcal{H}=1$ year $=8760 \mathrm{~h}$. The results of the relax factor shows quit high values as only one decision is taken for the whole year. This high relax factor has an impact on the LPSP which shows that, for example, during 2005 , only $77 \%$ of the whole time was supplied by the system. Nevertheless, the infrastructure was able to give $94 \%$ of the energy produced to the demand during the year. It means that the difference between the load and the provided production is not big. Also, one can see that the level of autonomy $L A$ is around $60 \%$ for all the years and that the unused energy is quite different from a year to another.

After analyzing the results, taking only one decision for a whole year is actually not accurate due to the uncertainty of the weather conditions. Then, we decided to shorten the resolution window to 1 week (52 resolutions for 1 year), 3 days (121 resolutions for 1 year) and to 1 day ( 365 resolutions for 1 year). The resulting metric are summerized respectively in the tables 456 
In the three tables, one can see that the smaller is the resolution window, the smaller is the relax factor. In fact, one can see that for the years 2004, 2005, 2006, rf decreased from around 0.08 to 0 which improved the loss of power supply and made it capable to answer to the data center demand more often. Contrarily, one can see that the percentage of the energy produced to demand PEP degrades with the smaller time windows. In fact, this is due to the inability of the system to answer to the demand in some time steps (i.e., instead of treating the lack of overproduction during a year, it is treated in a day). Indeed, the LPSP values computed for the different horizon $\mathcal{H} \in\{168,72,24\}$ are quite similar. Nevertheless, the standard deviation shows that the smaller is the resolution time window, the bigger is the periodic variation. To conclude, following to the metric results, the constraints of IT scheduling and the uncertainties of the weather prediction, the best resolution time windows is equal to 3 days as showed in Figure 2.

Based on Figure 2, one can see that around the hour 1000, the system is unable to meet the data center demand Pload: The fuel cell started working and the level of hydrogen decreases till reaching $0 \mathrm{~kg}$. In this moment, the Pload is relaxed by $r f$ for those three days. This relaxation could also be translated by a purchase of hydrogen to fulfill the demand. Starting from the hour 4000 (beginning of june), one can see that the level of hydrogen increases till reaching it maximum level at around the hour 5500. Pprod is then bigger than the demand. This unused renewable energy could be translated by selling hydrogen.

The resolution time of the MILP is in the order of seconds. For the one year time windows, the resolution time is equal to $32.48 \mathrm{~s}$. The weekly resolutions takes around $40.5 \mathrm{~s}$. The three days resolution time is equal to $44.8 \mathrm{~s}$ and the daily resolution time is equal to $55.7 \mathrm{~s}$.

\section{Conclusion and perspectives}

In this article, we presented first an analysis of the main trade offs involved in powering data centers or stand alone infrastructure with hybrid renewable energy system. we also presented the ANR DATAZERO project, in which this work take part, that aims to provide a data center with only renewable energy. Second, from the models used for HRES, the problem statement and the constraint satisfaction problem due to it, was settled. Third, a mixed integer linear program is deduced from the CSP in order to to manage a hybrid renewable energy system presenting four optimization scenarios depending on the resolution time window. These problems have been illustrated by several simulations. The optimal model is convenient to address the power commitment of an $100 \%$ renewable energy data center, since the resolution is obtained within few seconds.

\section{Acknowledgment}

This work was partly supported by the French Research Agency under the project Datazero (ANR-15-CE25-0012) and the EIPHI Graduate School (contract "ANR-17-EURE-0002").

\section{References}

1 Yasser Al-Saleh. Renewable energy scenarios for major oil-producing nations: The case of Saudi Arabia. Futures, 41(9):650-662, 2009.

2 Alain Billionnet. Optimisation discrète, De la modélisation à la résolution par des logiciels de programmation mathématique. 2007.

3 Tudor Cioara, Ionut Anghel, Marcel Antal, Sebastian Crisan, and Ioan Salomie. Data center optimization methodology to maximize the usage of locally produced renewable energy. In Sustainable Internet and ICT for Sustainability (SustainIT), 2015, pages 1-8. IEEE, 2015. 
4 Georges Da Costa. Heterogeneity: The key to achieve power-proportional computing. In 2013 13th IEEE/ACM International Symposium on Cluster, Cloud, and Grid Computing, pages 656-662. IEEE, 2013.

5 Weiqiang Dong, Yanjun Li, and Ji Xiang. Optimal sizing of a stand-alone hybrid power system based on battery/hydrogen with an improved ant colony optimization. Energies, 9(10):785, 2016.

6 Lee Fingersh, Dave Simms, Maureen Hand, Dave Jager, Jason Cotrell, Mike Robinson, Scott Schreck, and Scott M Larwood. Wind tunnel testing of NREL's unsteady aerodynamics experiment. In 20th ASME Wind Energy Symposium, 2001.

7 Mahdi Ghamkhari and Hamed Mohsenian-Rad. Energy and performance management of green data centers: A profit maximization approach. IEEE Transactions on Smart Grid, 4(2):1017-1025, 2013.

8 Inc. Gurobi Optimization. Gurobi optimizer reference manual, 2016.

9 Marwa Haddad, Jean-Marc Nicod, and Marie-Cecile Marion-Pera. Hydrogen infrastructure: Data-center supplyrefueling station synergy. In IEEE Vehicle Power and Propulsion Conference (VPPC'2017), 122017.

10 Marwa Haddad, Jean-Marc Nicod, Marie-Cécile Péra, and Christophe Varnier. Stand-alone renewable power system scheduling for a green data-center using integer linear programming. Technical report, mar 2019.

11 Nikos Hatziargyriou, Hiroshi Asano, Reza Iravani, and Chris Marnay. Microgrids. IEEE Power and Energy magazine, 5(4):78-94, 2007.

12 John K Kaldellis and Dimitris Zafirakis. The wind energy (r) evolution: A short review of a long history. Renewable energy, 36(7):1887-1901, 2011.

13 DEKD Kolokotsa, D Rovas, E Kosmatopoulos, and K Kalaitzakis. A roadmap towards intelligent net zero-and positive-energy buildings. Solar Energy, 85(12):3067-3084, 2011.

14 Khosrow Moslehi and Ranjit Kumar. A reliability perspective of the smart grid. IEEE Transactions on Smart Grid, 1(1):57-64, 2010.

15 Manajit Sengupta, Yu Xie, Anthony Lopez, Aron Habte, Galen Maclaurin, and James Shelby. The national solar radiation data base (NSRDB). Renewable and Sustainable Energy Reviews, 89:51-60, 2018.

16 Sunanda Sinha and SS Chandel. Review of recent trends in optimization techniques for solar photovoltaic--wind based hybrid energy systems. Renewable and Sustainable Energy Reviews, 50:755-769, 2015.

17 Jing $\mathrm{Xu}$ and Jose AB Fortes. Multi-objective virtual machine placement in virtualized data center environments. In 2010 IEEE/ACM Int'l Conference on Green Computing and Communications $\&$ Int'l Conference on Cyber, Physical and Social Computing, pages 179-188. IEEE, 2010. 
Table 1: Nomenclature

\begin{tabular}{|c|c|}
\hline Variable & Description \\
\hline $\mathcal{H}$ & A given time window $[h]$ \\
\hline$\Delta t$ & Interval of time between two time steps $[h]$ \\
\hline$k$ & Index for one time step within $\mathcal{H}$ \\
\hline$K$ & Number of time steps within $\mathcal{H}$ \\
\hline$P p v_{k}$ & $\mathrm{PV}$ power $[k W]$ \\
\hline$P w t_{k}$ & WT power $[k W]$ \\
\hline$S O C \max$ & Maximal state of Charge (SOC) $[k W h]$ \\
\hline SOCmin & Minimal SOC $[k W h]$ \\
\hline$S O C_{k}$ & SOC at instant $k \Delta t[k W h]$ \\
\hline$P c h_{k}$ & Power used to recharge battery $[k W]$ \\
\hline$P d c h_{k}$ & Power discharged from battery $[k W]$ \\
\hline LOHtarget $_{D}$ & $\mathrm{H}_{2}$ tank level targeted for a given day $D$ \\
\hline$L O H \max$ & $\mathrm{H}_{2}$ tank upper limit $[\%]$ \\
\hline$L H V h_{2}$ & $\mathrm{H}_{2}$ lower heating value $\left[\mathrm{kWh.} \mathrm{kg}^{-1}\right]$ \\
\hline$H H V h_{2}$ & $\mathrm{H}_{2}$ higher heating value $\left[k W h . \mathrm{kg}^{-1}\right]$ \\
\hline$L O H_{k}$ & $\mathrm{H}_{2}$ tank inventory level $[k g]$ \\
\hline$H_{2}$ & Maximal tank capacity $[\mathrm{kg}]$ \\
\hline Pezmax & Electrolyzer power upper limit [\%] \\
\hline Pezmin & Electrolyzer power lower limit [\%] \\
\hline $\mathrm{Pez}_{k}$ & Power put into electrolyzer $[k W]$ \\
\hline$Q e z_{k}$ & Electrolyzer $\mathrm{H}_{2}$ mass flow $[k g]$ \\
\hline Pfcmin & Minimum power to operate fuel cell $[k W]$ \\
\hline Pfcmax & Maximum power delivered by fuel cell $[k W]$ \\
\hline$P f c_{k}$ & Power delivered by fuel cell at period $k[k W]$ \\
\hline$Q f c_{k}$ & Fuel cell $\mathrm{H}_{2}$ mass flow $[k g]$ \\
\hline$\eta_{c h}$ & Battery charge efficiency [\%] \\
\hline$\eta_{d c h}$ & Battery discharge efficiency [\%] \\
\hline$\sigma$ & Battery self-discharge rate [\%] \\
\hline$\eta_{\text {tank }}$ & $\mathrm{H}_{2}$ tank efficiency $[\%]$ \\
\hline$\eta_{e z}$ & Electrolyzer efficiency [\%] \\
\hline$\eta_{f c}$ & Fuel Cell efficiency [\%] \\
\hline$\eta_{\text {inv }}$ & Inverter efficiency $[\%]$ \\
\hline$x_{k}$ & Battery in use $\left(x_{k}=1\right)$ or not $\left(x_{k}=0\right)$ \\
\hline$y_{k}$ & Electrolyzer in use $\left(y_{k}=1\right)$ or not $\left(y_{k}=0\right)$ \\
\hline
\end{tabular}

Table 2: Infrastructure sizing used in simulations

\begin{tabular}{|c|c|c|c|c|c|c|c|}
\hline Nwt & Npv & Pch & Pdch & SOCmax & Pez & Pfc & $\mathbf{H}_{2}$ \\
\hline 2 & 6650 & 980 & 555 & 5287 & 910 & 832 & 6382 \\
\hline
\end{tabular}


Table 3: Metric for a one year simulation with $\mathcal{H}=1$ year $=8760 h$

\begin{tabular}{|c|c|c|c|c|c|c|}
\hline \multirow{2}{*}{ year } & \multirow{2}{*}{ relaxation } & \multirow{2}{*}{ LA } & \multicolumn{2}{|c|}{ LPSP } & \multirow{2}{*}{ URE } & \multirow{2}{*}{ PEP } \\
\cline { 4 - 7 } & & & Average & SD & & \\
\hline 2004 & 0.07 & 0.67 & 0.98 & 0 & 3.45 & 0.93 \\
\hline 2005 & 0.08 & 0.69 & 0.77 & 0 & 84.94 & 0.94 \\
\hline 2006 & 0.11 & 0.66 & 0.85 & 0 & 47.08 & 0.90 \\
\hline 2007 & 0.31 & 0.64 & 0.75 & 0 & 82.54 & 0.77 \\
\hline 2008 & 0.18 & 0.64 & 0.85 & 0 & 43.84 & 0.78 \\
\hline 2009 & 0.27 & 0.63 & 0.83 & 0 & 62.16 & 0.78 \\
\hline 2010 & 0.48 & 0.60 & 0.72 & 0 & 121.51 & 0.67 \\
\hline 2011 & 0.43 & 0.64 & 0.66 & 0 & 155.09 & 0.73 \\
\hline 2012 & 0.3 & 0.61 & 0.79 & 0 & 60.94 & 0.76 \\
\hline
\end{tabular}

Table 4: Metric for a one year simulation with $\mathcal{H}=1$ week $=168 h$

\begin{tabular}{|l|l|l|l|l|l|l|}
\hline \multirow{2}{*}{ year } & \multirow{2}{*}{ relaxation } & \multirow{2}{*}{ LA } & \multicolumn{2}{|c|}{ LPSP } & \multirow{2}{*}{ URE } & \multirow{2}{*}{ PEP } \\
\cline { 4 - 5 } & & & Average & SD & & \\
\hline 2004 & 0 & 1 & 0.06 & 0.04 & 84.36 & 0.67 \\
\hline 2005 & 0 & 1 & 0.05 & 0.04 & 158.81 & 0.69 \\
\hline 2006 & 0 & 1 & 0.05 & 0.03 & 129.46 & 0.67 \\
\hline 2007 & 0.06 & 0.93 & 0.20 & 0.31 & 56.70 & 0.64 \\
\hline 2008 & 0.02 & 0.97 & 0.18 & 0.29 & 56.89 & 0.64 \\
\hline 2009 & 0.04 & 0.95 & 0.26 & 0.35 & 43.21 & 0.63 \\
\hline 2010 & 0.10 & 0.89 & 0.31 & 0.39 & 45.65 & 0.60 \\
\hline 2011 & 0.08 & 0.91 & 0.26 & 0.37 & 85.30 & 0.64 \\
\hline 2012 & 0.12 & 0.93 & 0.30 & 0.37 & 1.55 & 0.61 \\
\hline
\end{tabular}

Table 5: Metric for a one year simulation with $\mathcal{H}=3$ days $=72 \mathrm{~h}$

\begin{tabular}{|l|l|l|l|l|l|l|}
\hline \multirow{2}{*}{ year } & \multirow{2}{*}{ relaxation } & \multirow{2}{*}{ LA } & \multicolumn{2}{|c|}{ LPSP } & \multirow{2}{*}{ URE } & \multirow{2}{*}{ PEP } \\
\cline { 4 - 5 } & & & Average & SD & & \\
\hline 2004 & 0 & 1 & 0.06 & 0.05 & 84.59 & 0.67 \\
\hline 2005 & 0 & 1 & 0.05 & 0.05 & 159.24 & 0.69 \\
\hline 2006 & 0 & 1 & 0.05 & 0.05 & 129.81 & 0.67 \\
\hline 2007 & 0.04 & 0.95 & 0.17 & 0.27 & 54.05 & 0.64 \\
\hline 2008 & 0.02 & 0.98 & 0.15 & 0.24 & 57.52 & 0.64 \\
\hline 2009 & 0.04 & 0.95 & 0.20 & 0.30 & 42.42 & 0.63 \\
\hline 2010 & 0.08 & 0.91 & 0.28 & 0.37 & 44.27 & 0.59 \\
\hline 2011 & 0.06 & 0.93 & 0.22 & 0.33 & 85.46 & 0.64 \\
\hline 2012 & 0.05 & 0.94 & 0.23 & 0.32 & 1.55 & 0.61 \\
\hline
\end{tabular}


Table 6: Metric for a one year simulation with $\mathcal{H}=1$ day $=24 h$

\begin{tabular}{|l|l|l|l|l|l|l|}
\hline \multirow{2}{*}{ year } & \multirow{2}{*}{ relaxation } & \multirow{2}{*}{ LA } & \multicolumn{2}{|c|}{ LPSP } & \multirow{2}{*}{ URE } & \multirow{2}{*}{ PEP } \\
\cline { 4 - 5 } & & & Average & SD & & \\
\hline 2004 & 0 & 1 & 0.07 & 0.09 & 84.13 & 0.67 \\
\hline 2005 & 0 & 1 & 0.05 & 0.06 & 158.37 & 0.69 \\
\hline 2006 & 0 & 1 & 0.06 & 0.07 & 129.09 & 0.66 \\
\hline 2007 & 0.03 & 0.96 & 0.09 & 0.26 & 53.61 & 0.64 \\
\hline 2008 & 0.01 & 0.98 & 0.08 & 0.20 & 56.71 & 0.64 \\
\hline 2009 & 0.02 & 0.97 & 0.09 & 0.25 & 40.72 & 0.63 \\
\hline 2010 & 0.06 & 0.93 & 0.08 & 0.34 & 43.98 & 0.60 \\
\hline 2011 & 0.05 & 0.94 & 0.08 & 0.29 & 84.65 & 0.64 \\
\hline 2012 & 0.03 & 0.96 & 0.1 & 0.28 & 1.55 & 0.61 \\
\hline
\end{tabular}

